\title{
Comparison of Dimensional Accuracy of Three Different Impression Materials Using Three Different Techniques for Implant Impressions: An In Vitro Study
}

\author{
Shabab A Khan ${ }^{1}$, Sunita Singh ${ }^{2}$, Nudrat Neyaz ${ }^{3}$, Mishan Mohohar Jaiswal ${ }^{4}$, Aditi S Tanwar ${ }^{5}$, Amitu Singh $^{6}$
}

\begin{abstract}
Aim: To compare the dimensional accuracy of polyether, polyvinyl siloxane, and polyether polyvinyl siloxane hybrid impression materials using closed tray, open tray splinted, and open tray nonsplinted coping impression techniques and to find out the best suitable impression material and technique combination for implant impressions.

Materials and methods: The sample size of the study was 45 . The combinations of materials and techniques were divided into nine groups, namely polyvinyl siloxane with closed tray, polyvinyl siloxane with open tray nonsplinted copings, polyvinyl siloxane with open tray splinted copings, polyether with closed tray, polyether with open tray nonsplinted copings, polyether with open tray splinted copings, polyvinyl siloxane-polyether hybrid with closed tray, polyvinyl siloxane-polyether hybrid with open tray nonsplinted copings, and polyvinyl siloxanepolyether hybrid with open tray splinted copings. Five samples of each group were evaluated. A total of 45 impressions were taken. A stainless steel master metal framework with three internal hex implants was fabricated and used to generate the samples. A common condensation silicone putty spacer was applied over the impression copings attached to the implants, which was then duplicated. All trays were fabricated on this duplicated silicone die using light cure acrylic resin. Trays to be used for open tray techniques had their top removed for gaining access to screws of the impression copings. Splinting of coping for OS group was done using pattern resin. Impressions were poured, and master cast was generated. Interimplant distance was measured using stereomicroscope and an image analyzing software.

Results: Open nonsplinted technique resulted in significantly better replication of implant positions compared to open splinted and closed techniques. Hybrid impression material depicted significantly better dimensional accuracy than polyether and polyvinyl siloxane. Hybrid open nonsplinted depicted least mean difference in interimplant distance, followed by hybrid open splinted and polyether open splinted groups. Polyvinyl siloxane closed tray showed maximum difference in interimplant distance in comparison with other groups.

Conclusion: Polyvinyl siloxane-polyether hybrid impression material and open nonsplinted technique gave best replication of implant positions on the master cast.

Clinical significance: Accuracy of impressions and casts is of great importance for the fabrication and long-term clinical success of precisely fitting implant-retained prostheses.

Keywords: Dimensional accuracy, Impression materials, Impression techniques.

The Journal of Contemporary Dental Practice (2021): 10.5005/jp-journals-10024-3014
\end{abstract}

\section{INTRODUCTION}

Major advancements have been made in recent years in the rehabilitation of edentulous patients, the most promising being dental implants. Accuracy of impressions and casts is of great importance for the fabrication of precisely fitting implantretained prostheses and consequently for the long-term clinical success of these restorations. ' To achieve a passive fit, accurate positioning of implant replicas in the master cast must be assured. Factors that might influence the final positioning of implant replicas include impression technique (transfer or pickup), type of tray, impression material, and splinting (or not) of the implant copings. ${ }^{2-5}$

Two basic impression techniques commonly used for the transfer of implant positions from intraoral situation to a working cast are direct (open tray) and indirect (closed tray) techniques. ${ }^{6}$ In the direct technique, transfer copings remain in the impression and have to be unscrewed before impression can be removed from the mouth, whereas in the indirect technique, transfer copings are retained on the implants upon removal of the impression and have to be repositioned in their respective imprints of the impression. ${ }^{7}$ \begin{tabular}{l}
\hline \hline NIMS University Department of Prosthodontics, Crown \& Bridge, \\
Jaipur, Rajasthan, India \\
2,3,5 Pediatric and Preventive Dentistry, Patna, Bihar, India \\
${ }^{4}$ Department of Dentistry, Nalanda Medical College and Hospital, \\
Patna, Bihar, India \\
${ }^{6}$ Pediatric and Preventive Dentistry, Vananchal Dental College and \\
Hospital, Garhwa, Jharkhand, India \\
Corresponding Author: Mishan Mohohar Jaiswal, Department of \\
Dentistry, Nalanda Medical College and Hospital, Patna, Bihar, India, \\
Phone: +91 7004388816, Email : mishan.jaiswal@gmail.com \\
How to cite this article: Khan SA, Singh S, Neyaz N, et al. Comparison \\
of Dimensional Accuracy of Three Different Impression Materials Using \\
Three Different Techniques for Implant Impressions: An In Vitro Study. \\
J Contemp Dent Pract 2021;22(2):172-178. \\
Source of support: Nil \\
Conflict of interest: None \\
\hline \hline
\end{tabular}

Literature shows conflicting results regarding which technique provides better results. 
Several in vitro investigations have investigated the role of splinting the transfer copings to increase the accuracy of the direct impression technique. Theoretically, the rigid union of the transfers would increase the precision of casts by avoiding the movement of copings during various steps of impression making. ${ }^{8}$ The results of studies on this topic have also been inconsistent. ${ }^{9-11}$

Not only the impression technique, but the impression material per se has also been a keen topic of research with respect to dimensional accuracy of implant impression. Polyether $(\mathrm{PE})$ is a viable option due to its inherent rigidity and hydrophilicity. But there are some limitations of PE like poor tear strength, possibility of developing allergic reaction, and short working time. Besides, it gets stiff on setting and causes problem in removing the impression from the mouth. Polyvinyl siloxane (PVS), due to its additional flexibility, excellent dimensional accuracy, and good flow properties is recommended in cases of deep bony undercuts. ${ }^{13-14}$ But it has also some limitations like short working time and requirement of expensive equipment for performing procedure. A new material, a hybrid of polyvinyl siloxane and polyether (SENN, GC America, USA) combining their best features like high tear strength, high hydrophilicity, and excellent flow, is a recent addition to the class of implant impression materials that was introduced in 2005 to $2006 .^{12}$ Very few studies have been conducted to compare these three impression materials. Thus, a study to compare the dimensional accuracy of PE, PVS, and Hy impression materials using closed tray, open tray, and splinted coping impression technique was undertaken to find out the best suitable impression material and technique combination.

\section{Materials and Methods}

The study was conducted at the Department of Prosthodontics at Buddha Institute of Dental Sciences, Patna. A total of 45 samples were included in this study for evaluation.

\section{Master Metal Framework Fabrication}

A rectangular stainless steel master metal framework of dimensions $2 \times 3 \times 3 \mathrm{~cm}$ was milled out of a solid block. Three holes of diameter $3.8 \mathrm{~mm}$ and depth $10 \mathrm{~mm}$ were drilled into the block in an equilateral triangle manner with center of each whole being $10 \mathrm{~mm}$ away from the center of the other two. Three internal hex implants of dimensions $3.75 \times 10 \mathrm{~mm}$ were fixed to the metal die using molten field alloy. Four triangular notches were made on each corner of the framework for uniform orientation of the impression trays (Figs 1 and 2)

\section{Special Tray Fabrication}

A common internal spacer of $2 \mathrm{~mm}$ thickness was made using condensation silicone putty adapted around three close tray copings on the master die and the whole assembly duplicated in duplicating silicone. Special trays were fabricated on this duplicated silicone die using light cure acrylic resin. Each tray was cured for 7 minutes in the curing unit. Forty-five such trays were fabricated, and out of them, fifteen were left unmodified to be used for closed tray impression technique, while the remaining thirty had their roofs removed to provide access for impression copings in open tray impression technique, in which fifteen were used for splinted technique and fifteen for nonsplinted technique.

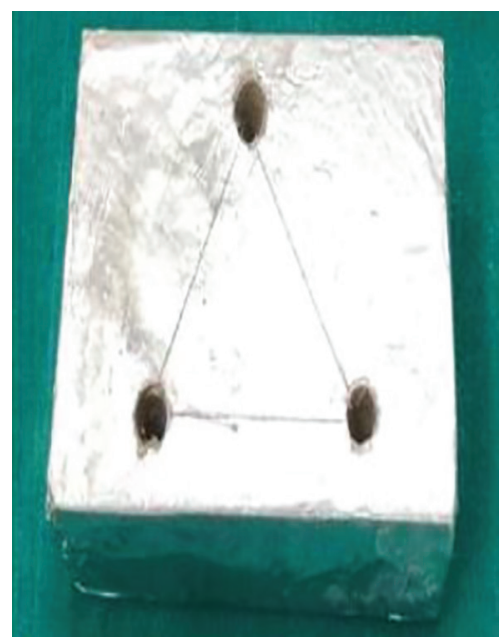

Fig. 1: Master metal block with three holes for implant placement

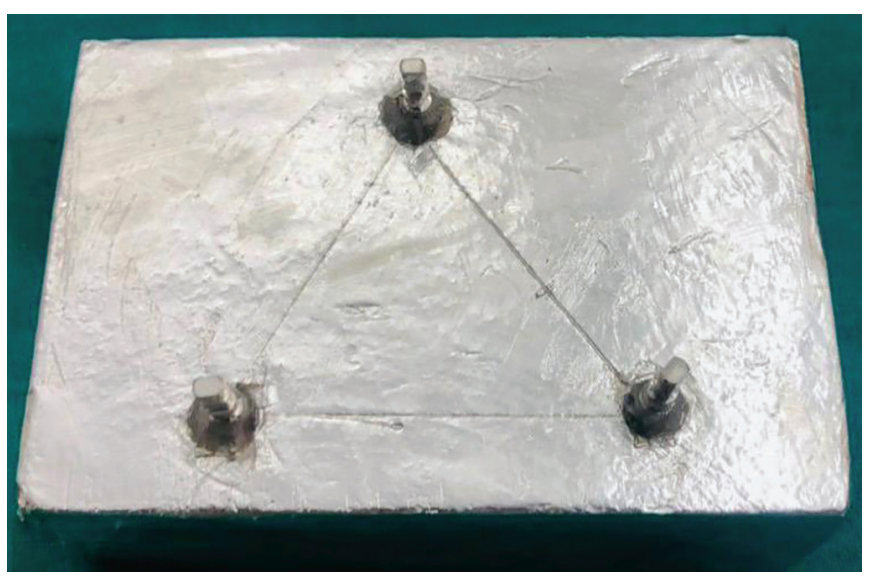

Fig. 2: Three implants in master metal framework

\section{Splinting Techniques}

The procedure of splinting was carried out by tying dental floss and joining the copings with autopolymerizing pattern resin. The splints were then sectioned using carborundum disks before the resin set completely and resplinted after setting with some more pattern resin in order to counteract the forces generated by polymerization shrinkage. Pattern resin exhibits very less polymerization shrinkage, thus minimizing any unwanted movement of impression copings by the procedure of splinting per se.

\section{Impression Techniques}

Impressions were divided into nine groups with five impressions each. Three impression techniques were evaluated. In each technique, 15 impressions were taken. Technique 1 used indirect (closed tray) (Fig. 3) impression technique with closed tray transfer copings. Technique 2 used direct (open tray) impression technique with nonsplinted copings. Technique 3 used direct (open tray) impression technique with splinted copings. Impressions for each technique were prepared with PVS (Dentsply Aquasil), PE (Dentsply Aquasil), and Hy impression materials (SENN, GC America, USA). Impressions were divided into nine groups. 


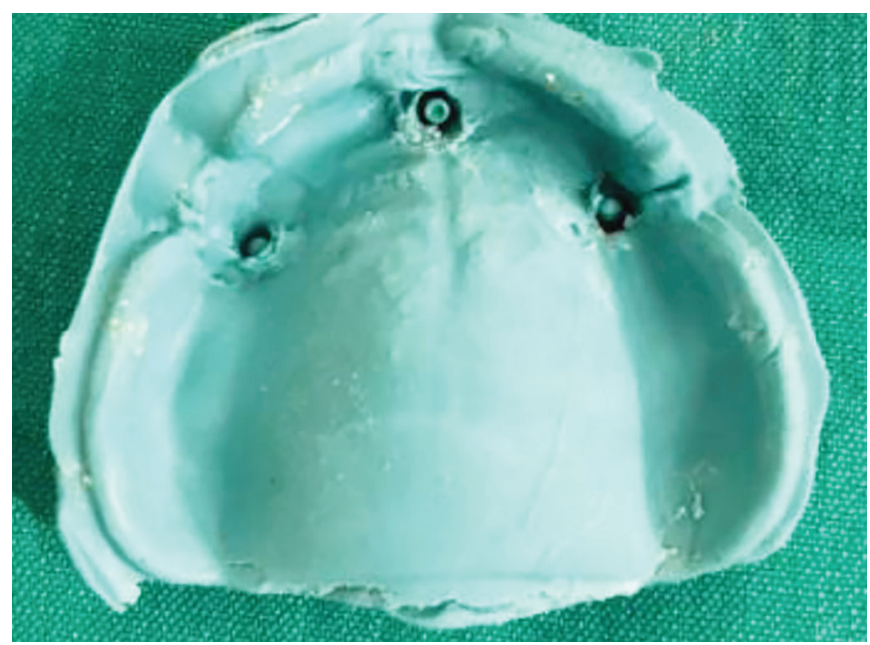

Fig. 3: Closed tray impression

- Gp PVS-C: Polyvinyl siloxane with closed tray. A total of five impressions were taken.

- Gp PVS-ONS: Polyvinyl siloxane with open tray nonsplinted copings. A total of five impressions were taken.

- Gp PVS-OS: Polyvinyl siloxane with open tray splinted copings. A total of five impressions were taken.

- Gp PE-C: Polyether with closed tray. A total of five impressions were taken.

- Gp PE-ONS: Polyether with open tray nonsplinted copings. A total of five impressions were taken.

- Gp PE-OS: Polyether with open tray splinted copings. A total of five impressions were taken.

- Gp Hy-C: PVS-PE hybrid with closed tray. A total of five impressions were taken.

- Gp Hy-ONS: PVS-PE hybrid with open tray nonsplinted copings. A total of five impressions were taken.

- Gp Hy-OS: PVS-PE hybrid with open tray splinted copings. A total of five impressions were taken.

\section{Master Cast Fabrication}

The impressions were beaded and boxed. Type IV dental stone $(80 \mathrm{gm})$ was mixed with distilled water $(20 \mathrm{ml})$, poured into each impression under vibrations, and allowed to set for 2 hours, according to manufacturer's instructions. For closed tray technique, the impression was pulled away from the cast and the impression copings were unscrewed. For open tray technique, the impression copings were first unscrewed and the impression was then easily pulled away.

\section{Dimensional Measurement}

A stereo microscope at $7 \times$ magnification was used to check the position of implant analogues in the master cast. The three corners of the hex of the three implant analogues were used as standard reference point for all the measurements. The three corners were marked as points $A, B$, and $C$. The interimplant distance was measured by measuring the distance between $A B, B C$, and $C A$. The three measured lengths were marked as $L 1, L 2$, and $L 3$. An additional measurement in the form of the perpendicular drawn over the side BC was recorded as $L 4$. The average of these four values was calculated and recorded as average interimplant distance. The abovementioned four dimensions were also measured on the master metal framework and used as control value. The average interimplant distance was then subtracted from the average interimplant distance on the master metal framework, and the mean difference of the average interimplant distance was calculated. This mean difference was the parameter on which all the statistical tests were applied. All the measurements were done using image analyzer software (Material Plus 4.2) by the same single investigator who was completely unaware of the study design.

\section{Statistical Analysis}

The data were analyzed using SPSS (Statistical Package for Social Sciences, Chicago, IL, USA) version 16.0. One-way analysis of variance followed by post hoc Bonferroni test was applied on all the three techniques and the three materials, namely PVS, PE, and $\mathrm{Hy}$. Level of significance was considered at $5 \%$ ( $p$-value $<0.05)$. The combined dimensional accuracy of the materials and techniques was compared using two-way analysis of variance. Level of significance was considered at $5 \%$ ( $p$-value $<0.05)$.

\section{Results}

The mean difference of average interimplant distance using closed tray technique, open tray nonsplinted, and open tray splinted technique with various impression materials is depicted in Table 1. The difference was found to be statistically significant. A similar pattern of distribution was noted in all the three techniques. The mean difference of Hy impression material was the least when compared to PVS and PE, thus demonstrating its superior dimensional accuracy irrespective of the technique used. It was also noted that PE gave better results than PVS.

Comparison within the various groups for mean difference of average interimplant distance using the three techniques with various materials was done using post hoc Bonferroni test. While using closed tray technique, PE was found to be significantly better than PVS, while Hy impression material was found to be significantly better than both PVS and PE. When using open tray nonsplinted technique, Hy worked significantly better than both PE and PVS. On the other hand, while using open tray splinted techniques, both $\mathrm{Hy}$ and PE worked significantly better than PVS.

The mean difference of average interimplant distance using various materials was compared using the ANOVA test, and the difference was found to be statistically significant for all the three impression materials. The mean difference was least for open tray nonsplinted technique with the use of both PVS and Hy impression

Table 1: Mean, standard deviation, and test of significance of mean difference of average interimplant distance with various materials

\begin{tabular}{llllll}
\hline Technique & & $N$ & Mean & Std. deviation & Sig. \\
\hline Closed tray & PVS & 5 & 0.06 & 0.007 & $0.000^{*}$ \\
& PE & 5 & 0.04 & 0.008 & \\
Open tray nonsplinted & Hy & 5 & 0.03 & 0.004 & \\
& PVS & 5 & 0.04 & 0.004 & $0.000^{*}$ \\
& PE & 5 & 0.03 & 0.008 & \\
Open tray splinted & Hy & 5 & 0.01 & 0.005 & \\
& PVS & 5 & 0.05 & 0.008 & $0.000^{*}$ \\
& PE & 5 & 0.02 & 0.005 & \\
& Hy & 5 & 0.02 & 0.007 & \\
\hline
\end{tabular}


Table 2: Mean, standard deviation, and test of significance of mean difference of average interimplant distance using impression material with various techniques

\begin{tabular}{|c|c|c|c|c|c|}
\hline Impression material & Technique & $N$ & Mean & Std. deviation & Sig. \\
\hline \multirow{3}{*}{$\begin{array}{l}\text { Polyvinyl siloxane } \\
\text { (PVS) }\end{array}$} & Closed tray & 5 & 0.06 & 0.007 & \multirow[t]{3}{*}{$0.001 *$} \\
\hline & $\begin{array}{l}\text { Open tray } \\
\text { nonsplinted }\end{array}$ & 5 & 0.04 & 0.004 & \\
\hline & $\begin{array}{l}\text { Open tray } \\
\text { splinted }\end{array}$ & 5 & 0.05 & 0.008 & \\
\hline \multirow[t]{3}{*}{ Polyether (PE) } & Closed tray & 5 & 0.04 & 0.008 & \multirow[t]{3}{*}{$0.007^{*}$} \\
\hline & $\begin{array}{l}\text { Open tray } \\
\text { nonsplinted }\end{array}$ & 5 & 0.03 & 0.008 & \\
\hline & $\begin{array}{l}\text { Open tray } \\
\text { splinted }\end{array}$ & 5 & 0.02 & 0.005 & \\
\hline \multirow{3}{*}{$\begin{array}{l}\text { PVS-polyether } \\
\text { hybrid (Hy) }\end{array}$} & Closed tray & 5 & 0.03 & 0.004 & \multirow[t]{3}{*}{$0.008^{*}$} \\
\hline & $\begin{array}{l}\text { Open tray } \\
\text { nonsplinted }\end{array}$ & 5 & 0.01 & 0.005 & \\
\hline & $\begin{array}{l}\text { Open tray } \\
\text { splinted }\end{array}$ & 5 & 0.02 & 0.007 & \\
\hline
\end{tabular}

*Statistically significant

materials, while for $\mathrm{PE}$, open tray splinted technique gave best results (Table 2). The use of closed tray technique gave maximum difference between average interimplant distances and thus resulted in least dimensional accuracy among the three techniques.

Further, within the group, comparison revealed that with regard to PVS, there was a statistically significant difference between closed tray and the open tray nonsplinted techniques, while no significant difference was found among the two other combinations. On the other hand, while using $\mathrm{PE}$, a statistically significant difference of dimensional accuracy with respect to closed tray was observed in comparison with open tray techniques, but there was no statistically significant difference between open tray nonsplinted and open tray splinted groups. In the same context when comparing Hy impression material, there was a statistically significant difference between closed tray and open tray nonsplinted groups, but no difference was seen between the other two groups (Table 3).

The ANOVA test was used to compare the mean difference of average interimplant distance using various impression techniques irrespective of the material used. A statistically significant difference was noted among the three techniques, with open tray nonsplinted technique depicting the least and closed tray technique the maximum mean difference from the master metal framework (Table 4).

When comparing the mean difference of average interimplant distance using various impression materials, irrespective of the technique used, a statistically significant difference was evident among the three materials, with Hy material depicting the least and PVS material the maximum mean difference from the master metal framework (Table 5).

For comparing the mean difference of average interimplant distance of various techniques and materials, a two-way ANOVA test was used. There was no statistically significant difference when the combined comparison of materials and techniques was done, although the combination of Hy-open tray nonsplinted group depicted best results, followed by Hy-open tray splinted and PE-open tray splinted groups. The combination of PVS material and closed tray technique showed maximum difference in the interimplant distance in comparison with other groups (Tables 6 and 7).
Table 3: Comparison within the various groups for mean difference of average interimplant distance using different impression materials with various techniques using the post hoc comparison (Bonferroni test)

\begin{tabular}{llll}
\hline Material & Techniques & Techniques & Sig. \\
\hline Polyvinyl silox- & Closed tray & Open tray nonsplinted & $0.001^{*}$ \\
ane (PVS) & & Open tray splinted & 0.050 \\
& Open tray & Closed tray & $0.001^{*}$ \\
& nonsplinted & Open tray splinted & 0.117 \\
& Open tray splinted & Closed tray & 0.050 \\
& & Open tray nonsplinted & 0.117 \\
Polyether (PE) & Closed tray & Open tray nonsplinted & $0.037^{*}$ \\
& & Open tray splinted & $0.008^{*}$ \\
& Open tray & Closed tray & $0.037^{*}$ \\
& nonsplinted & Open tray splinted & 1.000 \\
& Open tray splinted & Closed tray & $0.008^{*}$ \\
PVS-polyether & Closed tray & Open tray nonsplinted & 1.000 \\
hybrid (Hy) & & Open tray nonsplinted & $0.007^{*}$ \\
& Open tray & Open tray splinted & 0.147 \\
& nonsplinted & Closed tray & $0.007^{*}$ \\
& Open tray splinted & Closed tray & 0.379 \\
& & Open tray nonsplinted & 0.379 \\
\hline
\end{tabular}

*Statistically significant

Table 4: Mean, standard deviation, and test of significance of mean difference of average interimplant distance using various techniques

\begin{tabular}{lllll}
\hline & $n$ & Mean & Std. deviation & Sig. \\
\hline Closed tray & 15 & 0.04 & 0.015 & $0.005^{*}$ \\
Open tray nonsplinted & 15 & 0.03 & 0.012 & \\
Open tray splinted & 15 & 0.03 & 0.014 & \\
\hline
\end{tabular}

*Statistically significant

Table 5: Mean, standard deviation, and test of significance of mean difference of average interimplant distance using various materials

\begin{tabular}{lllll}
\hline & $n$ & Mean & Std. deviation & Sig. \\
\hline PVS & 15 & 0.05 & 0.011 & $0.000^{*}$ \\
PE & 15 & 0.03 & 0.012 & \\
Hy & 15 & 0.02 & 0.008 & \\
\hline
\end{tabular}

*Statistically significant

Table 6: Mean and standard deviation of mean difference of average interimplant distance using various impression materials and techniques

\begin{tabular}{llll}
\hline Materials & Techniques & Mean & Std. deviation \\
\hline PVS & Closed tray & 0.06 & 0.01 \\
& Open tray nonsplinted & 0.04 & 0.00 \\
& Open tray splinted & 0.05 & 0.01 \\
PE & Closed tray & 0.04 & 0.01 \\
& Open tray nonsplinted & 0.03 & 0.01 \\
& Open tray splinted & 0.02 & 0.01 \\
Hy & Closed tray & 0.03 & 0.00 \\
& Open tray nonsplinted & 0.01 & 0.01 \\
& Open tray splinted & 0.02 & 0.01 \\
\hline
\end{tabular}


Table 7: Test of significance of mean difference of average interimplant distance using various impression materials and techniques using univariate analysis of variance

\begin{tabular}{llllrl}
\hline \multicolumn{1}{l}{ Source } & $\begin{array}{l}\text { Type III sum } \\
\text { of squares }\end{array}$ & DF & Mean square & \multicolumn{1}{l}{ F } & Sig. \\
\hline Corrected model & 0.009 (a) & 8 & 0.001 & 23.573 & 0.000 \\
Intercept & 0.051 & 1 & 0.051 & 1112.244 & 0.000 \\
Materials & 0.006 & 2 & 0.003 & 65.756 & 0.000 \\
Techniques & 0.002 & 2 & 0.001 & 24.927 & 0.000 \\
Material & 0.000 & 4 & $8.22 \times 10^{-5}$ & 1.805 & 0.149 \\
techniques & & & & & \\
Error & 0.002 & 36 & $4.56 \times 10^{-5}$ & & \\
Total & 0.061 & 45 & & & \\
Corrected total & 0.010 & 44 & & & \\
\hline
\end{tabular}

\section{Discussion}

Achieving a passive fit ensures long-term success of the implant, which in turn is dependent on several factors including dental impression recorded, physical and mechanical properties of impression material, impression technique employed, selection of the impression tray, die material's accuracy, machining forbearance of prosthetic components, depth, and angulation of implant placement. ${ }^{15-17}$ An implant can be deemed to have a passive fit when all the surfaces of implant and prosthesis are aligned without force application and the gap formed between metallic framework and implants is within limits established by science $(111 \mu \mathrm{m}) .^{18}$

The fidelity of the impression with respect to dimensional stability is critical as even small discrepancies can result in difficulty while the restoration is fabricated and delivered. Hence, a study to compare the dimensional accuracy among PE, PVS, and Hy impression materials using open tray, closed tray, and splinted coping impression techniques was undertaken, to determine the best technique and impression material. Our study showed that open tray nonsplinted technique resulted in significantly better replication of implant positions when compared to the other techniques and the Hy impression material depicted significantly better dimensional accuracy than PE and PVS.

When using closed tray and open tray nonsplinted techniques, Hy impression material worked significantly better than the other two materials, whereas for open tray splinted technique, both Hy and PE were equally effective. With the use of PVS and Hy impression materials, open tray nonsplinted technique gave the best results, while for $\mathrm{PE}$, open tray splinted technique works best. The combination of Hy impression material and open tray nonsplinted technique depicted the least mean difference in interimplant distance, followed by Hy-OS and PE-OS groups. The combination of PVS and closed tray technique showed maximum difference in the interimplant distance in comparison with other groups, showing least dimensional accuracy.

Papaspyridakos et al. investigated the role of splinted versus nonsplinted implant impression techniques on the accuracy of fit of fixed implant prostheses in edentulous patients and concluded that splinted impression techniques generated more accurate implant impressions and master cast. ${ }^{19}$ Keerthna et al. compared the accuracy of implant cast by splinting and nonsplinting techniques in open tray method in angulated implants and found a significant difference among the groups, suggesting splinted impression technique to be efficacious for multiple angled implants. ${ }^{20}$
Elshenavy et al. studied the dimensional accuracy of casts obtained from three impression techniques (indirect, unsplinted direct, and acrylic resin-splinted direct technique) for lower casts with implants at different angulations and reported that acrylic resin-splinted direct technique produced the most accurate casts, followed by direct nonsplinted and indirect techniques. ${ }^{21}$ Jalalian et al. analyzed splinted and nonsplinted open-tray impression techniques on different angled implants and suggested splinted impression technique to be superior for angulated implants. ${ }^{22}$ Arora et al. evaluated the accuracy of implant casts generated with splinted and nonsplinted impression techniques with multiple parallel and nonparallel implants and observed that splinted impression technique exhibited higher accuracy. ${ }^{23}$ The findings of all these studies were not in concordance with our study, as our study showed open tray nonsplinted technique to provide better results. Selvaraj et al. performed an in vitro study to evaluate the accuracy of master cast using open tray impression technique with conventional (autopolymerizing acrylic resin) and novel splinting materials (bis-GMA) and concluded that master cast obtained by either splinting material exhibited no difference from the reference model, a finding negating ours.

Shankar et al. in their in vitro study investigated the accuracy of open and closed tray impression techniques with three impression materials, namely PVS, PE, and vinyl siloxanether [VSE], on angulated implants and summarized that VSE impression material yielded more accurate casts than those of PVS and PE, a finding similar to ours. Moreover, lowest mean deviation was found in casts made from open tray technique splinted with floss and resin. ${ }^{24}$ Kankane et al. compared the accuracy of direct and indirect impression techniques using PVS and PE impression materials and reported the combination of direct technique with PE impression material to show the highest level of dimensional accuracy. ${ }^{25}$ Our study also showed similar results.

Ebadian et al. determined the effect of PVS and PE impression materials and techniques on the dimensional accuracy of implant definitive casts. They found no statistically significant difference between open and closed tray impression techniques; however, less distortion and deviation were observed in open tray technique. ${ }^{26}$ Vojdani et al. compared the accuracy of three impression materials, namely PE, PVS, and vinyl siloxanether, with open tray technique in parallel and nonparallel implant positions. They deduced that in parallel conditions, type of impression material did not affect the accuracy of implant impressions; however, in nonparallel conditions, PVS was a better choice followed by vinyl siloxanether and PE. ${ }^{27}$ The findings of these two studies were not in accordance with our study.

Reddy et al. evaluated the dimensional accuracy of casts made from PVS and PE impression materials in parallel and angulated implants. They showed both the materials had similar dimensional accuracy for transfer procedures. ${ }^{28}$ On the other hand, in our study, PE showed better results as compared to PVS. Naik et al compared the three-dimensional accuracy of open and closed impression technique to transfer the intraoral position of implant fixture to the working cast using PE, PVS, and condensation silicone. They commended open tray impression technique along with PE and PVS impression materials for making implant impressions to obtain accurate results, a finding similar to ours. ${ }^{29}$

Gupta et al. evaluated dimensional accuracy of master casts obtained using different impression trays (custom or stock trays) and materials (PE, vinyl siloxanether) with open tray impression technique and found $\mathrm{PE}$ to be more accurate than vinyl siloxanether, a finding not similar to ours. Moreover, 
they also recommended rigid nonperforated stock trays as an alternative for custom trays for multi-implant impressions when used with medium viscosity impression materials. Balouch et al. compared the dimensional accuracy between open and closed tray implant impression techniques in $15^{\circ}$ angled implants and showed closed tray impression technique to be more accurate. ${ }^{30}$ The finding of this study was not in concordance with our study, as our study showed open tray technique to show statistically significant results in comparison with closed techniques. Tandon et al. evaluated and compared the effect of closed tray and open tray impression techniques with PVS and PE as impression materials on the dimensional accuracy of implant definitive casts. They reported either of the techniques and material could be used to make implant definitive casts, as they did not show any statistically significant difference, a finding not similar to ours. ${ }^{31}$

Baig MR analyzed 59 studies on different aspects of multi-unit implant impression accuracy. Fifteen studies compared PVS and PE, and 11 found no difference between the two materials in terms of impression accuracy. Thirty studies analyzed the splint effect, 13 found splinting better, and 13 others elicited no difference between splinting and nonsplinting. Among the 25 studies examining pickup and transfer impression techniques, 12 favored pickup over transfer and 11 found no differences between the two. They thus concluded that PVS and PE were the preferred impression materials for multi-unit implant impressions. The evidence on splinting was inconclusive, and data supporting splint to nonsplint were neutral. Pickup was the better performing technique than transfer, especially with increased number of implants. ${ }^{32}$

The limitations of this study were that there was no analysis of any discrepancy that may have taken place in vertical direction during process of implant impression recording. There was analysis of casts only in the x-axis, $y$-axis, and z-axis. There was also no analysis of nonparallel conditions of the implants. Besides, this was an in vitro study, and therefore, the results may be different from the condition that may be present intra-orally. Therefore, more in vivo studies should be conducted.

\section{Conclusion}

Within the constraints of this study, it can be considered that PVS-PE hybrid impression material in combination with open tray nonsplinted technique gave best replication of the implant positions on the master cast.

\section{References}

1. Lee $H$, Ercoli $C$, Funkenbusch PD, et al. Effect of subgingival depth of implant placement on the dimensional accuracy of the implant impression: an in vitro study. J Prosthet Dent 2008;99(2):107-113. DOI: 10.1016/S0022-3913(08)60026-8.

2. Cabral LM, Guedes CG. Comparative analysis of 4 impression techniques for implants. Implant Dent 2007;16(2):187-194. DOI: 10.1097/ID.0b013e3180587b3f

3. Burns J, Palmer R, Howe $L$, et al. Accuracy of open tray implant impressions: an in vitro comparison of stock versus custom trays. J Prosthet Dent 2003;89(3):250-255. DOI: 10.1067/mpr.2003.38.

4. Rashidan N, Alikhasi M, Samadizadeh S, Beyabanaki E, Kharazifard MJ. Accuracy of implant impressions with different impression coping types and shapes. Clin Implant Dent Relat Res. 2012 Apr;14(2):218-225. DOI: 10.1111/j.1708-8208.2009.00241.x.

5. VigoloP, Fonzi F,MajzoubZ, etal.An evaluation of impression techniques for multiple internal connection implant prostheses. J Prosthet Dent 2004;92(5):470-476. DOI: 10.1016/j.prosdent.2004.08.015.
6. de Avila ED, Barros LA, Del' Acqua MA, et al. Comparison of the accuracy for three dental impression techniques and index: an in vitro study. J Prosthodont Res 2013;57(4):268-274. DOI: 10.1016/j.jpor.2013.07.001.

7. Conrad HJ, Pesun IJ, DeLong R, Hodges JS. Accuracy of two impression techniques with angulated implants. J Prosthet Dent. 2007 Jun;97(6):349-356. DOI: 10.1016/S0022-3913(07)60023-7.

8. Branemark PI, Zarb GA, Albrektsson T. Tissue-integrated prostheses. Osseointegration in clinical dentistry. Chicago: Quintessence; 1985, pp. 11-76.

9. Siadat $\mathrm{H}$, Alikhasi M, Beyabanaki E, Rahimian S. Comparison of Different Impression Techniques When Using the All-on-Four Implant Treatment Protocol. Int J Prosthodont. 2016 May-Jun;29(3):265-270. DOI: $10.11607 /$ ijp. 4341.

10. Burawi G, Houston F, Byrne D, et al. A comparison of the dimensional accuracy of the splinted and unsplinted impression techniques for the Bone-Lock implant system. J Prosthet Dent 1997;77(1):68-75. DOI: 10.1016/s0022-3913(97)70209-9.

11. Hsu CC, Millstein PL, Stein RS. A comparative analysis of the accuracy of implant transfer techniques. J Prosthet Dent 1993;69(6):588-593. DOI: 10.1016/0022-3913(93)90287-x.

12. Enkling $\mathrm{N}$, Bayer $\mathrm{S}$, Jöhren $\mathrm{P}$, et al. Vinylsiloxanether: a new impression material. clinical study of implant impressions with vinylsiloxanether versus polyether materials. Clin Implant Dent Relat Res 2012;14(1):144-51. DOI: 10.1111/j.1708-8208.2009.00240.x

13. Gupta S, Narayan Al, Balakrishnan D. In Vitro Comparative Evaluation of Different Types of Impression Trays and Impression Materials on the Accuracy of Open Tray Implant Impressions: A Pilot Study. Int J Dent. 2017;2017:6306530. DOI: 10.1155/2017/6306530.

14. Akalin ZF, Ozkan YK, Ekerim A. Effects of implant angulation, impression material, and variation in arch curvature width on implant transfer model accuracy. Int J Oral Maxillofac Implants 2013;28(1):149-157. DOI: 10.11607/jomi.2070.

15. Wee AG. Comparison of impression materials for direct multi-implant impressions. J Prosthet Dent 2000;83(3):323-331. doi: 10.1016/s00223913(00)70136-3.

16. Baig MR. Accuracy of impressions of multiple implants in the edentulous arch: a systematic review. Int J Oral Maxillofac Implants. 2014 Jul-Aug;29(4):869-80. DOI: 10.11607/jomi.3233.

17. Saunders WP, Sharkey SW, Smith GM, et al. Effect of impression tray design upon the accuracy of stone casts produced from a singlephase medium-bodied polyvinyl siloxane impression material. J Dent 1992;20(3):189-192. DOI: 10.1016/0300-5712(92)90139-4.

18. Osman M, Abubakr NH, Suliman A, Ziada H. The impact of impression coping geometrical design on accuracy of implant impressions: an experimental study. Int J Implant Dent. 2020 Oct 10;6(1):54. DOI: 10.1186/s40729-020-00256-0.

19. Papaspyridakos P, Chen CJ, Gallucci GO, Doukoudakis A, Weber HP, Chronopoulos V. Accuracy of implant impressions for partially and completely edentulous patients: a systematic review. Int J Oral Maxillofac Implants. 2014 Jul-Aug;29(4):836-45. DOI: 10.11607/jomi.3625.

20. Selvaraj S, Dorairaj J, Mohan J, Simon P. Comparison of implant cast accuracy of multiple implant impression technique with different splinting materials: An in vitro study. J Indian Prosthodont Soc. 2016;16(2):167-75. DOI: 10.4103/0972-4052.167937.

21. Elshenawy EA, Alam-Eldein AM, Abd Elfatah FA. Cast accuracy obtained from different impression techniques at different implant angulations (in vitro study). Int J Implant Dent 2018;4(1):9. DOI: 10.1186/s40729-018-0118-6.

22. Buzayan M, Baig MR, Yunus N. Evaluation of accuracy of completearch multiple-unit abutment-level dental implant impressions using different impression and splinting materials. Int J Oral Maxillofac Implants. 2013 Nov-Dec;28(6):1512-1520. DOI: 10.11607/jomi.2958.

23. Arora A, Upadhyaya V, Parashar KR, et al. Evaluation of the effect of implant angulations and impression techniques on implant cast accuracy - an in vitro study. J Indian Prosthodont Soc 2019;19(2):149158. DOI: 10.4103/jips.jips_337_18.

24. Shankar YR, Sahoo S, Krishna MH, Kumar PS, Kumar TS, Narula S. Accuracy of implant impressions using various impression techniques 
and impression materials. J Dent Implant 2016;6(1):29-36. DOI: 10.4103/0974-6781.190384

25. Tabesh M, Alikhasi M, Siadat H. A Comparison of implant impression precision: Different materials and techniques. J Clin Exp Dent. 2018;10(2):e151-e157. DOI:10.4317/jced.54457

26. Nakhaei M, Madani AS, Moraditalab A, Haghi HR. Three-dimensional accuracy of different impression techniques for dental implants. Dent Res J 2015;12:431-437 DOI: 10.4103/1735-3327.166190

27. Vojdani M, Torabi K, Ansarifard E. Accuracy of different impression materials in parallel and nonparallel implants. Dent Res J 2015;12(4):315-222. DOI: 10.4103/1735-3327.161429.

28. Reddy S, Prasad K, Vakil H, et al. Accuracy of impressions with different impression materials in angulated implants. Niger J Clin Pract 2013;16(3):279-284. DOI: 10.4103/1119-3077.113447.

29. Naik J, Behera SSP, Galagali G, et al. Comparison of three dimensional accuracy of two implant level impression techniques; using three different elastomeric impression materials-an in vitro study. IP Ann Prosthodont Restor Dent 2019;5(3):68-71. DOI: 10.18231/j. aprd.2019.016.

30. De La Cruz JE, Funkenbusch PD, Ercoli C, Moss ME, Graser GN, Tallents $\mathrm{RH}$. Verification jig for implant-supported prostheses: A comparison of standard impressions with verification jigs made of different materials. J Prosthet Dent. 2002 Sep;88(3):329-336. DOI: 10.1067/ mpr.2002.128070.

31. Tandon A, Bulbule NS, Jagtap AK, Kakade DM. Comparative Evaluation of the Dimensional Accuracy of Closed Tray and Open Tray Impression Technique for Dental Implants using Two Different Impression Materials. J Clin Diagn Res 2018;12(11): ZC34-ZC38. DOI: 10.7860/ JCDR/2018/36235.12296.

32. Baig MR. Multi-unit implant impression accuracy: a review of the literature. Quintessence Int 2014;45(1):39-51. DOI: 10.3290/j. qi.a30769. 